\author{
Dolinsek, S.; Kopac, J.; Viharos, Zs. J.; Monostori, L.; An intelligent AE sensor for the monitoring \\ of finish machining process, The Second International Conference on Intelligent Processing and \\ Manufacturing of Materials, July 10-15, 1999, Honolulu, Hawaii, pp. 847-853.
}

\title{
AN INTELLIGENT AE SENSOR FOR THE MONITORING OF FINISH MACHINING PROCESS
}

\author{
S. Dolinšek*, J. Kopač*, Z.J. Viharos**, L. Monostori ** \\ *University of Ljubljana, Faculty of Mechanical Engineering, Ljubljana, Slovenia \\ ** Computer and Automation Research Institute, Hungarian Academy of Science, Budapest, Hungary
}

\begin{abstract}
The paper presents the latest results of sensing the cutting process on the basis of AE signals and some particularities in further development of the monitoring model for the finish turning process. Due to non-linearity, the large number of influencing parameters and missing information in AE data, the Artificial Neural Networks were chosen as a monitoring decision tool. The problem of accurateness in predicting the surface roughness on the basis of AE - because of the mutual interdependence of the data - requires a special procedure for building a neural network model. The final aim of such an approach is presented as improvements in learning or considerable reduction in error prediction. Further development of the monitoring model has the goal of building a so-called intelligent sensor, which should be able to perform the signal conditioning and feature extraction process.
\end{abstract}

\section{INTRODUCTION}

Most of the reports on research into the machining processes usually start with a similar ascertainment: the complexity of the cutting process is one of the main obstacles to successful modeling or monitoring of processes; this fact gives us the impetus to continue permanent investigations. There are no simple answers or quick solutions; a reliable monitoring approach or a complete control system for the cutting process is a task in which successful solutions could be obtained only through numerous, systematic investigations covering the different scientific areas incorporating sensor technologies, signal processing techniques, modeling methods, etc.

Probably one of the best of the latest reviews of such efforts has been made within the CIRP groups, where the conclusions stated that the different monitoring systems with acceptable commercial reliability are now available in the market, although the narrow range of performance provides only limited applicability (Byrne and others at [1]). The report also confirms one of the main gaps in this kind of research; i.e. to develop a system as an integrated part of an intelligent machine tool, much more should be done at both the hardware and software level to obtain a simple and reliable sensor for machining applications. At present - when the development of manufacturing processes heavily depends on information technologies the realistic process models are also one of the prerequisites for predicting the performance of metal cutting operations. The latest report on the modeling of machining operations (C.A. van Luttervelt and others at [2]) concluded, that most of the research deals with possible new ways of obtaining better control of machining operations; however a common framework is still missing.

At the first IPMM conference the monitoring concept on the basis of sensing Acoustic Emission signals in finish machining processes was presented (see Dolinsek at [3]). From the contents of the AE signals we were able to extract significant features from the process, depending on the cutting conditions, which serve as learning data for the ANN structure. The model should be applicable for practical cutting in such a way that the predicted values of surface roughness could be a sign to adapt the cutting parameters in order to achieve the required surface quality or to detect disturbances in the process (tool wear, unfavorable chip shape, lack of coolant). In the introduction we also draw attention to the lack of adequate sensors and indicate that the sensing technology will play an important role in the development of future manufacturing systems.

Further investigation of our monitoring concept for finish machining processes was therefore oriented towards the search for reliable sensing. Some of the results using the AE-jet sensor were discussed at SEM and CIRP conferences (see Dolinsek at [4] and [5]). The main advantages of this sensor were presented as improvements of the signal to noise ratio, simple upgrade, and the fact that the cutting process and sensor are not reciprocally disturbed. Through the spectral analysis technique, and 
with adequate averaging procedures, we were therefore able to gain some useful information for the further development of our monitoring model.

Artificial neural networks (ANNs) were used as a operating tool because they can handle strong non-linearites, a large number of parameters, missing information, and the characteristics of the data which are also significant in our monitoring approach. Based on their inherent learning capabilities, ANNs can adapt themselves to changes in the production environment, and can also be used in case where no detailed information is available about the relationships among the various manufacturing parameters. In many cases, there is also no exact knowledge about the relationships among parameters; it is unknown which input-output configuration of an ANN can satisfy the accuracy requirements. Therefore, a method is needed for automatic input-output configuration of the applied ANN model. This paper therefore addresses the problem of automatic input-output configuration and generation of ANN-based monitoring models, i.e. those parameters to be considered as inputs, and those as output, in order to accurately predict surface roughness and classify tool wear in the finish turning process.

\section{AE-JET SENSOR FOR MONITORING A FINISH MACHINING PROCESS}

In researching Tool Condition Monitoring (TCM) systems for the manufacturing processes and introducing them to the workshop environment we are engaged in solving three main tasks :

- building up a sensor system which is reliable for sensing the process parameters with minimal influence on the process,

- applying proper signal processing techniques capable of processing the real life signals,

- developing decision-making algorithms capable of estimating the process conditions.

In such an feature-based approach, we observe some features, extracted from sensor signals in order to identify different process conditions and compare them to normal and unfavorable cutting conditions. This process is generally not too complicated, but the success of the monitoring depends greatly on exact correlation of the measured parameters to the cutting process characteristics - i.e. the sensors are the first and main component leading to the successful solution of our tasks. Once we find or build-up a sensor which satisfies the main requirements demanded in practical monitoring approaches: measurement close to the machining point; no influences on the machine-tool characteristics; function independent of tool or workpiece; low costs; maintenance and wear free; resistance to dirt and to mechanical and thermal influences, minimal reciprocal disturbances between the process and sensor, simple upgrade which allows easy further improvements. Thus we can further develop our monitoring model by applying signal processing, feature extraction and decision making procedures. When this intelligent part is successfully solved the final hardware integration into the sensor is not a complicated task.

One of the most promising tool monitoring techniques is based on sensing the Acoustic Emission (AE) signals generated at the cutting zone. Extensive publications have demonstrated the extreme sensitivity of AE signals to certain process parameters (etc. Dornfeld at [6]). In general it is agreed that during metal cutting, plastic deformation (continuous type of AE signals) and fracture of the material (burst type of $\mathrm{AE}$ signals) are major sources for $\mathrm{AE}$ waves. One of the basic researches of the AE phenomena in the cutting is that made by Moriwaki, who illustrated in detail seven possible sources of the $\mathrm{AE}$ signals in the vicinity of the cutting process [7]. However in sensing and analyzing the AE signals generated in the cutting process we will always face two main obstacles:

- it is almost impossible to built-up a physical model of the AE signal in relation to the AE waves, since the signal generated in real cutting processes in complex workpiece structures is continuous and random,

- we expect from the sensor used in metal cutting problems a reliable sensing of AE signals from all sources of generation of the signal with the ability to differentiate between the particular sources, but without any other interference's.

Due to those limitations the most common approach of the application of the $\mathrm{AE}$ in monitoring of cutting processes is at present still a simplified sensing of the mixed AE signals. From the content of the acquired AE signals using a suitable postprocessing procedure one can then identify different process conditions. Although many different sensors are available for $\mathrm{AE}$ measurements, only few can be used in a machine tool in which aggressive ambient conditions occur. The main disadvantages of traducers, which are mainly designed for non-destructive inspection or research work, are that they cannot withstand the high temperatures, large coolant volumes and abrasive wear through chips. With a new concept of $\mathrm{AE}$ transducers (see [8]), - the Water-jet AE sensors - a liquid or coolant stream is used as a transmission medium to transfer AE signals generated from the cutting process to the PZT element. As the distance between the cutting zone and transducer element is small, the damping effect is minimized, and the signal-to-noise ratio is significantly improved. The construction of 
the sensor, developed for our monitoring task, presented in Fig 1, was a practically built-in CNC finish turning machine. The applicability of this sensor in the finish turning process was tested throughout the proper analysis of the acquired AE signals and further relation of their content to the process conditions.
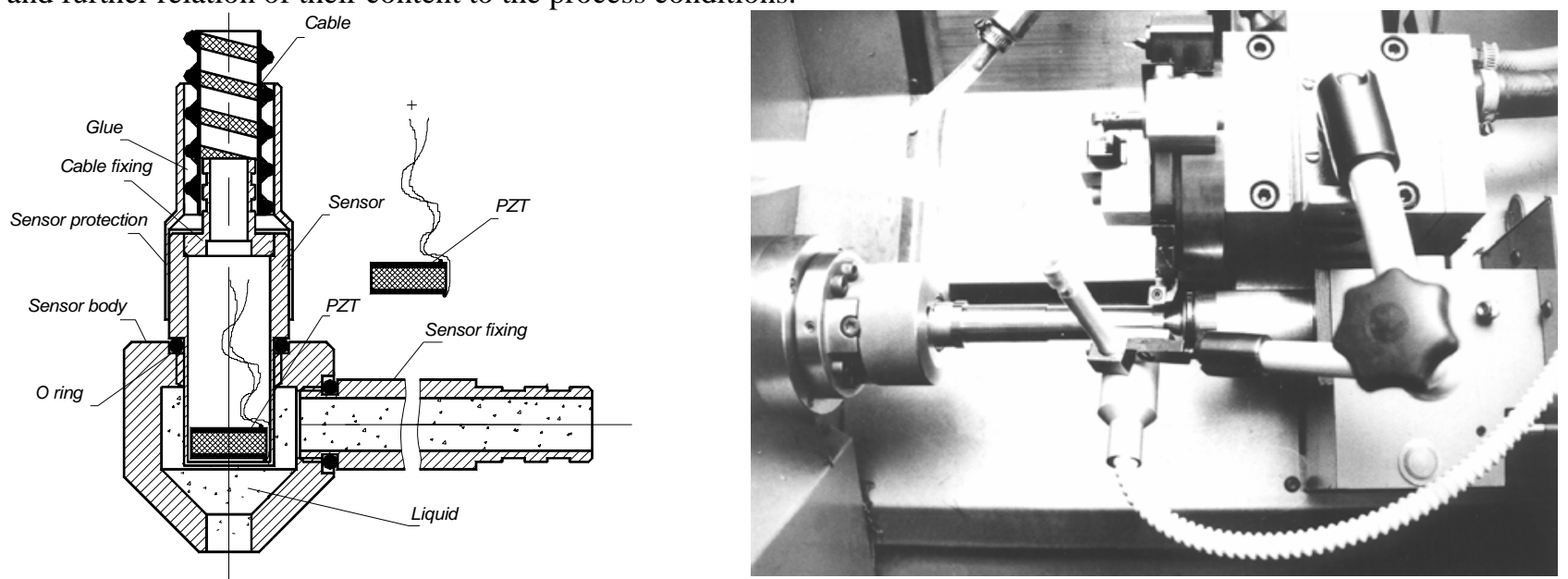

Fig. 1. AE-jet sensor for monitoring a finish machining process built-in CNC turning machine

All the tests were made by using cutting conditions producing continuous chips. Therefore, a frequency analysis of the signals - using the well known assumption for continuous random signals - could be applied as the signal processing technique. In order to obtain satisfactory amplitude estimations in spectral analysis, 75 averages within sample and 15 averages between the samples, were performed. Fig. 2 presents the response of the sensor when the tool is not cutting (free run of the turning machine). We can notice that the power spectra of the signals shows a distinctive amplitude peak in the range of the resonant frequency of the sensor, and that the energy of the signal is mainly distributed within the range of 100$610 \mathrm{kHz}$.
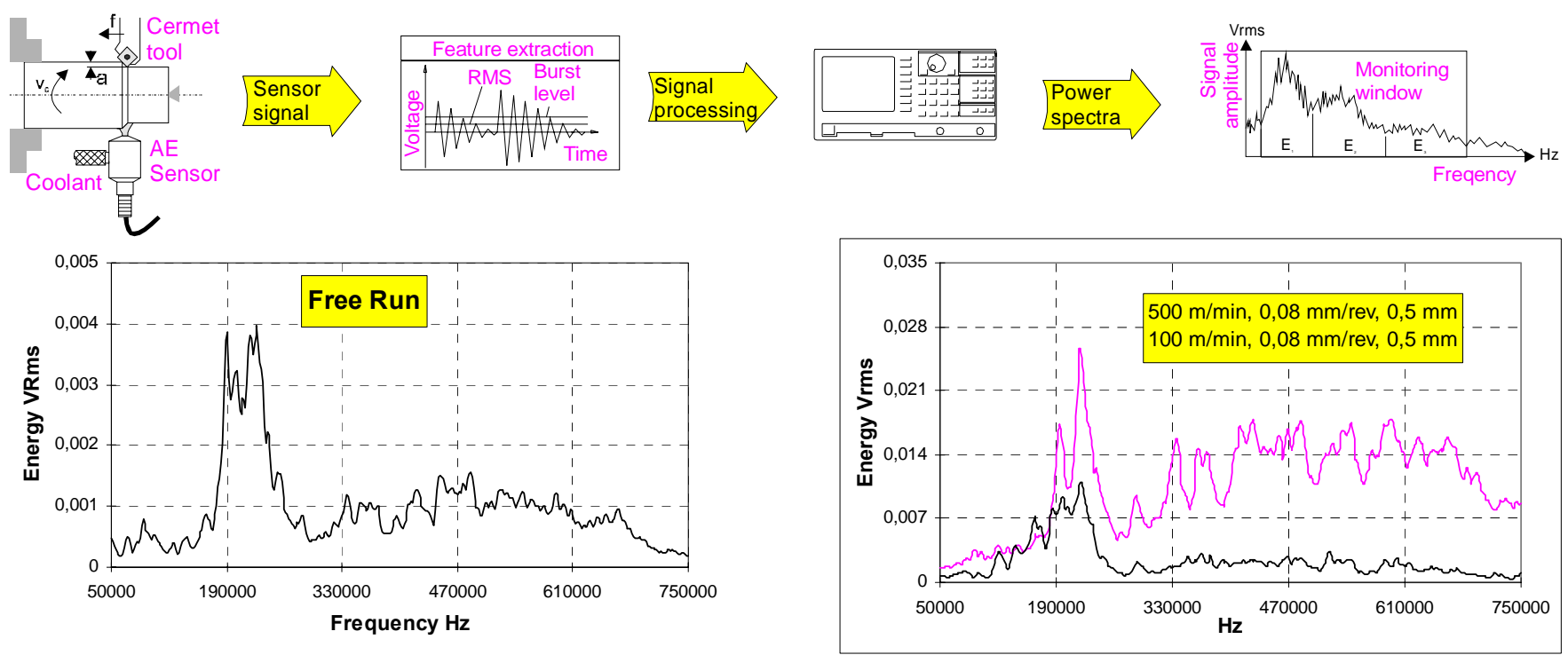

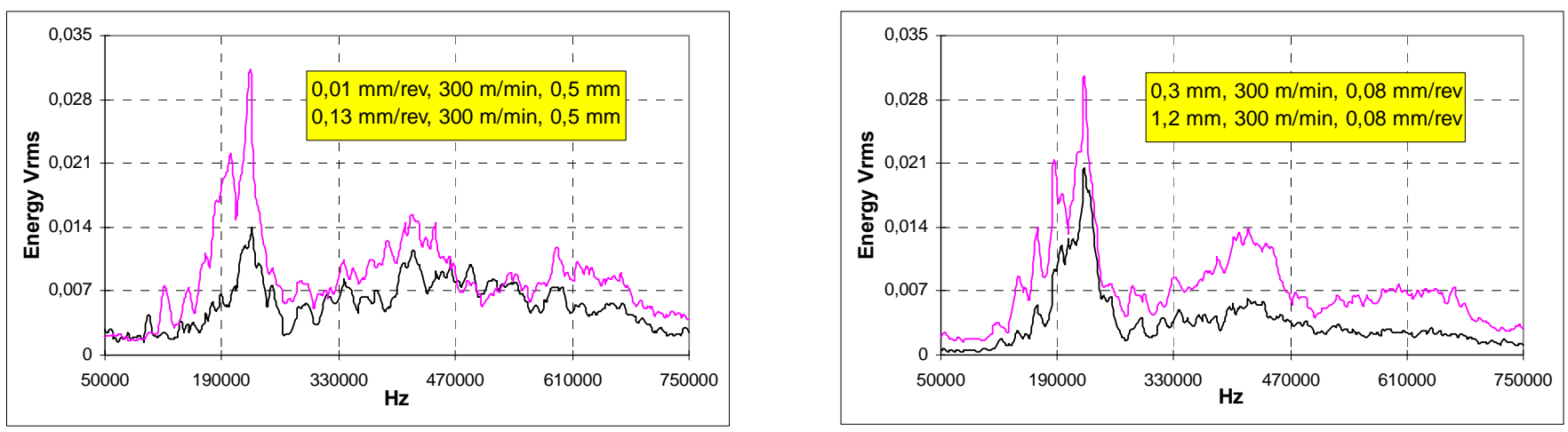

Fig. 2. Spectra of the AE signals in finish turning process obtained in cutting with different cutting conditions In Fig. 2, which also presents a comparison of the spectra of AE signals obtained in cutting with different parameters, we notice that the spectra and their energy are altered according to the changes in cutting conditions. From the results presented here, and those already published [4,9], the following conclusions can be drawn:

- the energy of the AE signal is mainly distributed in the frequency range of $100-610 \mathrm{kHz}$,

- the sensivity of the sensor depends on the position of the piezo-ceramics placed inside the coolant stream,

- this sensor can be used in workshop conditions with the coolant supplied by the machine tool pump,

- tool wear is one of the most influential factors in increasing the energy of the AE signal.

- the sensor is sensitive to AE signals obtained by cutting different workpiece materials and according to the variations of the machining parameters; their influence can be noticed from different spectral energies.

\section{ANN AS A TOOL FOR INTELLIGENT PROCESSING OF AE SIGNALS}

Considering the input and output variables in our model for monitoring a finish turning process as a set of parameters, the ANN model estimates a part of this parameter set based on the remaining part. This selection strongly influences the accuracy of the developed model, especially if dependencies between parameters are non-invertable. In different cutting conditions (e.g. first cut with sharp tool, non-uniformities in material structure, disturbances in coolant flow), the tasks are different; consequently, the estimation capabilities of the related applied models are different, even if the same set of parameters obtained with the same cutting parameters is used. One of the main goals of the research was to find a general model for a set of assignments which can satisfy the accuracy requirements. This goal was achieved by a sequential forward selection (SFS) search algorithm, which uses the heuristic of speed of ANN learning. This method incorporates:

- determination of the number of output variables,

- determination for every parameter to be input or output.

The method also builds up the appropriate ANN model without considering the given assignment of the engineer; it is also useful in the case of strong non-linear relationships. [10]. Research was also focused on how to apply the general model for various tasks. Usually, the engineer knows some parameters of a process and the modelling task is to determine the other parameters while satisfying some constraints. After obtaining the general ANN model, in almost every case a part of the input and of the output variables of the general model are known by the user; the task of the modelling is then to search for the remaining, unknown input and output parameters. IN order to realise this, the simulated annealing search method was used to determine the unknown input parameters. After obtaining the appropriate input parameters, the unknown output parameters can be determined by a simple ANN estimation. The values of the unknown input parameters are appropriate, if:

- they are between their minimum and maximum values,

- the estimated unknown output parameters are between their minimum values,

- the estimation of the output parameters determined on the basis of known and unknown input parameters ensures that the estimated values of the known output parameters are equal to their known values

The first and second conditions determine the validity of the ANN model. With the help of this method, all of the possible assignments of engineers can be solved by the general ANN model. [11]. As a practical demonstration of the method, an example of the evaluation of the AE signals from finish machining experiments will be analysed. In this investigation the 
target is to estimate the produced roughness of a surface based on known values of cutting parameters and measured AE signal. The cutting parameters, which were varied, are feed ( $\mathrm{f}=0,01-0,2 \mathrm{~mm} / \mathrm{rev})$, cutting speed $\left(\mathrm{v}_{\mathrm{c}}=100-500 \mathrm{~m} / \mathrm{min}\right)$ and depth of cut $(\mathrm{a}=0,1-1,2 \mathrm{~mm})$. From the measured AE signal and its energy content significant features were calculated using particular energies in four frequency ranges (according to the ascertainments from previous investigation, see [3,4]): 50-750 kHz, 99-249.5 kHz, 249.5-400 kHz and 400-610 kHz. These seven parameters together act as known parameters, while the roughness acts as parameter still to be estimated. Since the non-linear dependencies among these parameters are already experienced, an ANN model can consequently be used to realise the mapping among parameters.

The concept of building an intelligent sensor for a finish machining process is based on idea of performing the on-line learning process as fast as possible in the whole range of applicable cutting parameters with satsfactory accuracy in prediction of the process conditions. Using statistical planing of experiments, 21 one measurements were used to build up an ANN model (the selection of parameters are presented in Fig. 3) and seven machining situations to test the behaviour of the applied ANN model. The above-described method was used to demonstrate the automatic input-output configuration of the ANN.

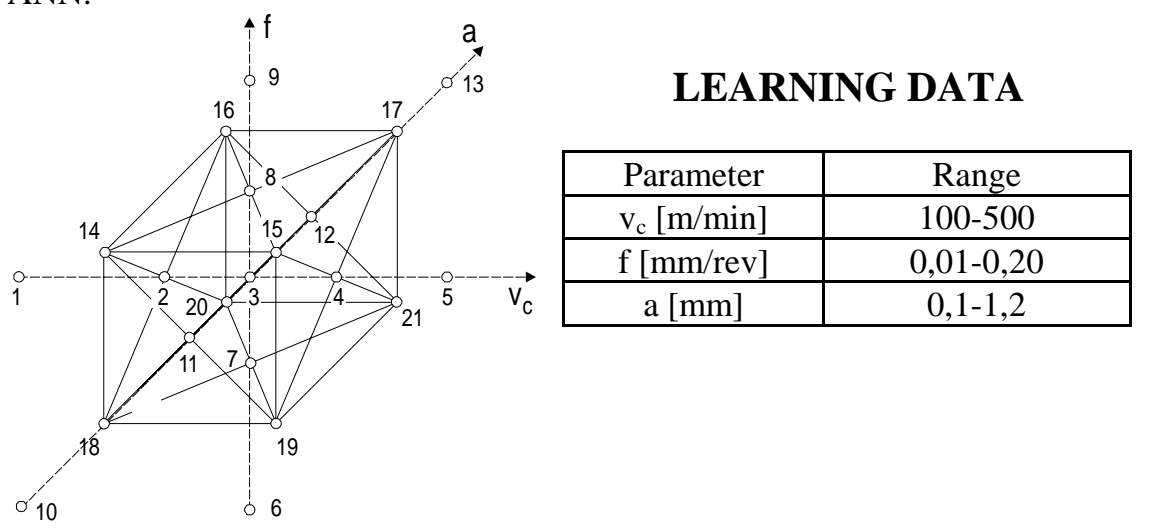

TEST DATA

\begin{tabular}{|c|c|c|c|}
\hline No. & $\mathrm{v}_{\mathrm{c}}$ & $\mathrm{f}$ & $\mathrm{a}$ \\
\hline 1 & 300 & 0,07 & 0,5 \\
\hline 2 & 200 & 0,07 & 0,5 \\
\hline 3 & 400 & 0,07 & 0,5 \\
\hline 4 & 300 & 0,04 & 0,5 \\
\hline 5 & 300 & 0,10 & 0,5 \\
\hline 6 & 300 & 0,07 & 0,2 \\
\hline 7 & 300 & 0,07 & 0,8 \\
\hline
\end{tabular}

Fig. 3. Planing of the experiment for learning and test data.

Because the number of learning vectors is small, we firstly tried to build up an ANN model with one hidden layer and two hidden nodes. The target average estimation accuracy of the ANN model was $\pm 2.5 \%$. The above described method found one output parameter that can be estimated by the ANN model based on the remaining parameters (inputs). This was the energy parameter E1 (50-750kHz). The roughness becomes the input of the model. Fig. 4 shows the input-output configuration of this ANN model. With the help of this new method it is therefore possible to estimate the unknown parameters based on the values of known parameters, regardless of whether were the input or output of the ANN model. In such a way, the estimation of the roughness based on known values of $\mathrm{v}, \mathrm{f}, \mathrm{a}, \mathrm{E}_{1}, \mathrm{E}_{2}, \mathrm{E}_{3}, \mathrm{E}_{4}$ was performed. The seven test situations were reviewed at the first stage. In each of the situations, the estimations were repeated ten times to check if there were more solutions for the given estimation task. The estimations of surface roughness with this ANN model, presented in Fig. 4, are not very accurate, but the developed algorithm reports through the parameter $E_{1}$ that the roughness can not be estimated accurately enough. 

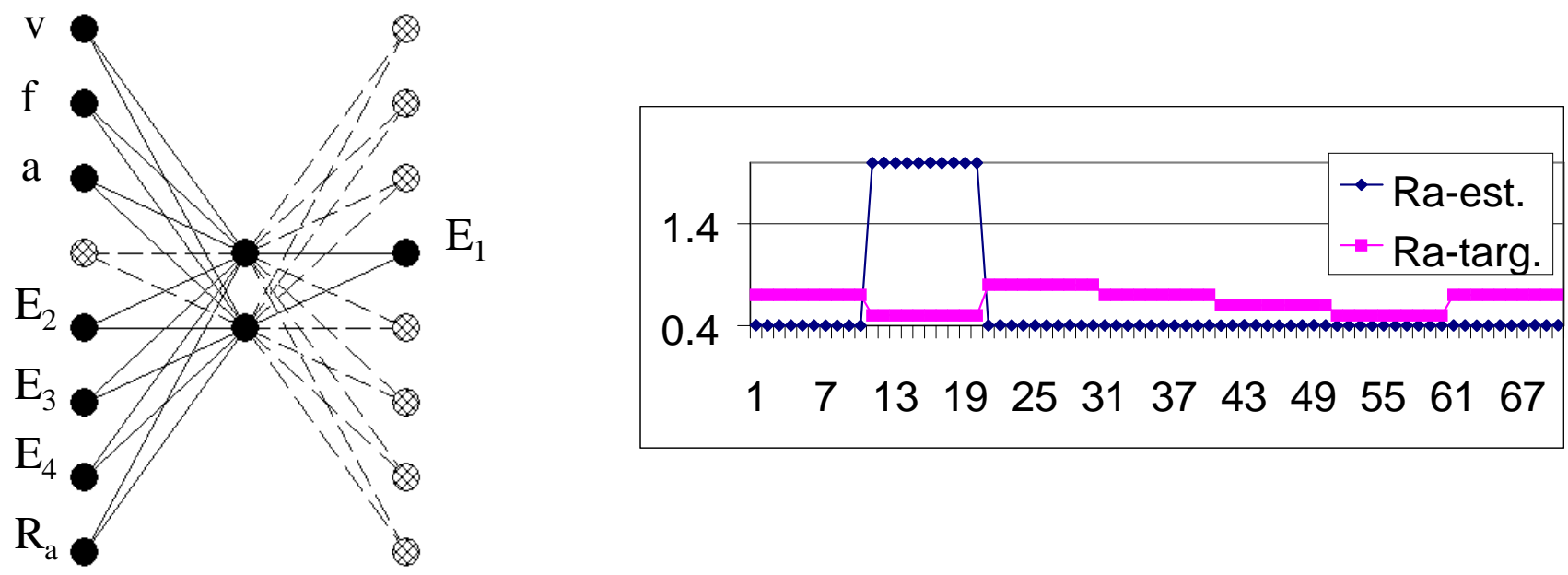

Fig. 4. The input/output configuration of the first ANN model and estimate and target roughness.

Very similar results of estimations were obtained with the situations used in building up the ANN model. The conclusion is that the ANN model is not appropriate; consequently, the number of input nodes needs to be enlarged. The second investigations were performed with an ANN having 6 hidden nodes. In this case the input-output configuration of the resulted ANN was different from the previous one. This ANN with five inputs and three outputs is presented in Fig. 5. The outputs are $E_{1}, E_{2}$, and $E_{4}$. To check if the model was sufficiently accurate, the roughness estimation was performed on the learning data set. The results of these estimations show that the ANN model learns the dependencies between the inputs and outputs from the learning data set. For testing the model in the test cutting situations, estimations of roughness were performed with this new ANN configuration, repeated ten times for each of the situations. The results of the estimated and the measured roughness (Fig. 5) show that the ANN is not able to estimate the roughness in these situations, yet this ANN model could perfectly estimate the learning data. This shows that in further investigation the quantity of learning data has to be enlarged.
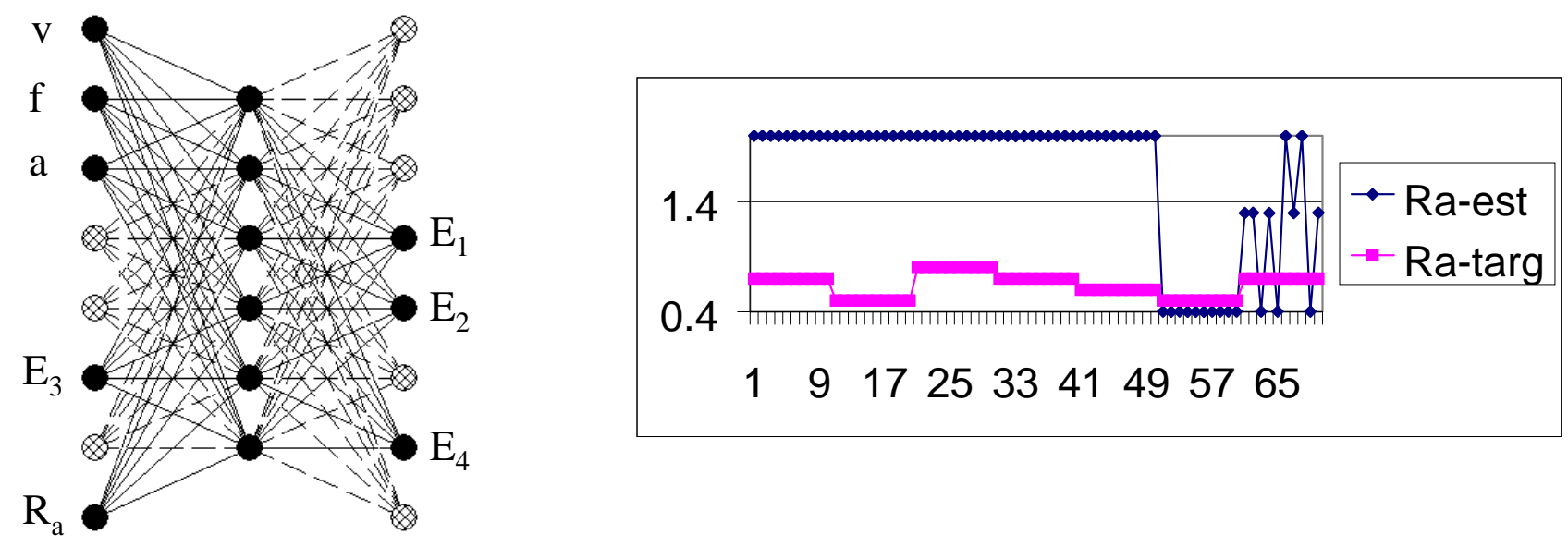

Fig. 5. The input/output configuration of the second ANN model and estimate and target roughness.

\section{CONCLUSIONS}

The introduction of automatic control of the machine tools, and the highly invisible cutting area inside the machine tool, have made the monitoring of the processes even more difficult. In recent years extensive efforts have been spent in developing such systems; however, reliable and marketable products are still not available. In the paper the latest results are presented in using a more sensitive sensor - the AE-jet sensor - to monitor the finish turning process in order to obtain the prescribed surface finish of the products. The response of the sensor is adequate to the damping characteristics of the cutting process in relation to the changes in cutting conditions; the response can be observed directly in spectral energies in different frequency 
ranges of the $\mathrm{AE}$ signal. Using the energies of the $\mathrm{AE}$ signals, obtained with cutting, under both the favorable and unfavorable conditions, we have built up a concept of monitoring decision-making. Artificial Neural Networks can handle non-linearities, multidimensionality, missing information and also the main problem in the AE results obtained, the overlap presented by the same values of roughness or AE for different cutting parameters. With the new method of building an ANN structure, which can determine the number of variables and search for input and output variables, the learning phase is shortened and the prediction is improved. The results obtained demonstrated a good direction for further work in this direction.

\section{REFERENCES}

1. G. Byrne, D. Dornfeld, I. Inasaki, G. Ketteler, W. König, R. Teti, 1995. Tool Condition Monitoring, The Status of Research and Industrial Application. Annals of the CIRP, 44(2), 24-41.

2. C.A. van Luttervelt, T.H.C. Childs, I.S. Jawahir, F. Klocke, P.K. Venuvinod, 1999. Present Situation and Future Trends in Modeling of Machining Operations. Annals of the CIRP, 48(2), 587-626

3. S. Dolinsek, 1997. Hybrid intelligent systems in monitoring of finishing machining processes, Australasia Pacific Forum on Intelligent Processing and Manufacturing of Materials. Brisbane, Australia.

4. S.Dolinsek, 1997. Tool Condition Monitoring Using AE-jet sensor, 1997. Postconference Procedings of the SEM Spring conference, SEM , 165-174.

5. S. Dolinsek, 1998. Monitoring of finish machining process using AE-jet sensor, $31^{\text {st }}$ CIRP International Seminar on Manufacturing Systems, Berkeley, USA, 540-545.

6. J.Liu, D. Dornfeld, 1996. ASME, Journal of Engineering for Industry, 118 (4), 199-207.

7. T. Moriwaki, 1983. Application of Acoustic Emission Measurement to Sensing of Wear and Breakage of Cutting Tool. Bull. Japan Soc. Prec. Eng., 17 (3), 154-160.

8. I. Grabec, W. Sachse, 1991. Automatic Modeling of physical phenomena, Application of ultrasonic data. Journal of Applied Physics, 69 (9), 24-32.

9. S.Dolinsek, J. Kopac, 1999. Acoustic emission signals for tool wear identification, $12^{\text {th }}$ International Conference on Wear of Materials, Atlanta, USA

10. Zs. J. Viharos, L. Monostori, S. Markos, 1999. Selection of input and output variables of ANN based modeling of cutting processes. CIRP Proceedings of the X. Workshop on Supervising and Diagnostics of Machining Systems, Poland.

11. Zs. J. Viharos, L. Monostori, 1999. Automatic input-output configuration and generation of ANN-based process models and its application in machining. Proceedings of the XII. International Conference on IEA/AIE Systems, Kairo, Egypt. 